ARTICLE

Received 15 Jun 2016 | Accepted 31 Oct 2016 | Published 19 Dec 2016

DOI: $10.1038 /$ ncomms13740

OPEN

\title{
Fluorination-enabled optimal morphology leads to over $11 \%$ efficiency for inverted small-molecule organic solar cells
}

Dan Deng ${ }^{1, \star}$, Yajie Zhang ${ }^{1, \star}$, Jianqi Zhang ${ }^{1}$, Zaiyu Wang ${ }^{2}$, Lingyun Zhu' ${ }^{1}$ Jin Fang ${ }^{1,3}$, Benzheng Xia ${ }^{1}$, Zhen Wang ${ }^{1}$, Kun Lu' ${ }^{1}$, Wei Ma ${ }^{2} \&$ Zhixiang Wei, ${ }^{1,3}$

Solution-processable small molecules for organic solar cells have attracted intense attention for their advantages of definite molecular structures compared with their polymer counterparts. However, the device efficiencies based on small molecules are still lower than those of polymers, especially for inverted devices, the highest efficiency of which is $<9 \%$. Here we report three novel solution-processable small molecules, which contain $\pi$-bridges with gradient-decreased electron density and end acceptors substituted with various fluorine atoms ( $\mathrm{OF}, 1 \mathrm{~F}$ and $2 \mathrm{~F}$, respectively). Fluorination leads to an optimal active layer morphology, including an enhanced domain purity, the formation of hierarchical domain size and a directional vertical phase gradation. The optimal morphology balances charge separation and transfer, and facilitates charge collection. As a consequence, fluorinated molecules exhibit excellent inverted device performance, and an average power conversion efficiency of $11.08 \%$ is achieved for a two-fluorine atom substituted molecule.

\footnotetext{
${ }^{1}$ CAS Key Laboratory of Nanosystem and Hierarchical Fabrication, CAS Center for Excellence in Nanoscience, National Center for Nanoscience and Technology, Beijing 100190, China. ${ }^{2}$ State Key Laboratory for Mechanical Behavior of Materials, Xi'an Jiaotong University, Xi'an 710049, China. ${ }^{3}$ University of Chinese Academy of Sciences, Beijing 100049, China. * These authors contributed equally to this work. Correspondence and requests for materials should be addressed to K.L. (email: Ivk@nanoctr.cn) or to W.M. (email: msewma@xjtu.edu.cn) or to Z.W. (email: weizx@nanoctr.cn).
} 
O rganic solar cells (OSCs) have attracted intense attentions due to their potential for solution processing cheap and flexible devices. Comparing with conventional device architectures, inverted devices exhibit improved environmental stability and more preferable for industrial applications ${ }^{1}$. Polymer solar cells with power conversion efficiency (PCE) higher than $10 \%$ are mainly obtained through inverted devices ${ }^{2-4}$, and the highest efficiency has reached $11.7 \%$ (ref. 5). Solution-processable small molecules for OSCs have attracted intense attentions for their advantages of high purity and definite molecular structures compared with polymers ${ }^{6-11}$. To date, the PCE based on small molecules has reached $10 \%$ by conventional devices ${ }^{8,12}$. However, attempts to develop inverted devices for small molecules are not as successful as that of polymers ${ }^{13-16}$, and the highest PCE reported is $8.84 \%$ (ref. 17 ), which is much lagging behind their polymer couterparts.

To obtain highly efficient OSCs, decisive parameters, namely, open circuit voltage $\left(V_{\mathrm{oc}}\right)$, fill factor $(\mathrm{FF})$ and short-circuit current $\left(J_{\text {sc }}\right)$ should be enhanced. Each parameter could be expressed as following general formula:

$$
\Lambda=\Lambda_{\mathrm{max}}-\Lambda_{\mathrm{loss}}\left(\Lambda=V_{\mathrm{oc}}, \text { FF or } J_{\mathrm{sc}}\right)
$$

A proved successful strategy in molecular design is to increase $\Lambda_{\max }$. The approaches involve increasing ionization potential of the donor $\left(\mathrm{IP}_{\mathrm{D}}\right)$, narrowing bandgap and enhancing mobility to achieve high $V_{\mathrm{oc}}, J_{\mathrm{sc}}$ and $\mathrm{FF}$, respectively ${ }^{18-20}$. However, $\mathrm{IP}_{\mathrm{D}}$ enhancement would lead to bandgap increase ${ }^{19}$, and $\left(V_{\mathrm{oc}} \times J_{\mathrm{sc}}\right)_{\max }$ is limited by Shockley-Queisser model. To further maximize PCE, an alternative strategy is to minimize $\Lambda_{\text {loss }}$. The main loss of $V_{\mathrm{oc}}$ is related to the disorder arrangement of donors/ acceptors and their poor contact with electrode ${ }^{21-23}$; the main losses of $\mathrm{FF}$ and $J_{\mathrm{sc}}$ are ascribed to the recombination induced by undesirable distribution of intermix and crystalline phases, interfacial traps and poor domain purity ${ }^{24,25}$. Consequently, all losses are related to the bulk-heterojunction $(\mathrm{BHJ})$ morphology, the optimization of which could simultaneously minimize $\Lambda_{\text {loss }}$ for all three parameters.

The adjustment of the donor-acceptor (D:A) interaction is an essential approach to optimize the $\mathrm{BHJ}$ morphologies. A difference in surface free energies between donors and acceptors would result in a repulsive interaction (lower miscibility), which acts as the internal driving force to form phase separation ${ }^{26,27}$. Hence, a proper disparity of surface free energies is significant to achieving optimized lateral morphology. Furthermore, a lower surface free energy of donors in comparison with acceptors would induce surface enrichments and vertical phase separation ${ }^{28}$, both of which could effectively decrease losses of $V_{\mathrm{oc}}, J_{\mathrm{sc}}$ and FF through suppression of recombination by modifying interface contact, forming charge-blocking regions and facilitating charge collection $^{24,28}$.

In this paper, three medium bandgap molecules are designed and synthesized with thiophene-substituted benzodithiophene (TBDT) as a core, 2-(thiophen-2-yl)thieno [3,2-b]thiophene as $\pi$-bridges and end-capped with $1 \mathrm{H}$-indene-1,3(2H)-dione, 4-fluoro-1H-indene-1,3(2H)-dione or 4,7-difluoro-1H-indene1,3(2H)-dione; these molecules are abbreviated as BTID-0F, BTID-1F and BTID-2F (Fig. 1a). With incremental introduction of fluorine to end-capped units, the PCE for inverted devices increases from $8.30 \%$ for BTID-0F to $10.4 \%$ for BTID-1F and to $11.3 \%$ for BTID-2F. A hierarchical morphology with higher domain purity, enhanced surface enrichment and more directional vertical phase distribution is induced by fluorination, thereby $V_{\mathrm{oc}}, J_{\mathrm{sc}}$ and FF are increased simultaneously.

\section{Results}

Design and characterization of small molecules. To benefit the charge carrier transport, a two-dimensional donor unit, TBDT was selected as the core ${ }^{29}$. A new $\pi$-bridge, namely, 2-(thiophen2-yl)thieno[3,2- $b]$ thiophene, was designed with two advantages: first, it introduces stronger aromaticity units of thieno $[3,2-b]$ thiophene to increase $\mathrm{IP}_{\mathrm{D}}$, aiming to increase attainable $V_{\text {oc }}$. Second, it presents an inner gradient-decreased electron density distribution (Supplementary Fig. 1), benefited for backbone hole transfer. To lower the miscibility and increase dielectric constant, $1 \mathrm{H}$-indene-1,3(2H)-dione was selected as acceptor because of its stronger polarity and aromatic difference in comparison with [6,6]-phenyl- $\mathrm{C}_{71}$-butyric acid methyl ester $\left(\mathrm{PC}_{71} \mathrm{BM}\right)^{26}$. To decrease molecular surface free energies and miscibility with $\mathrm{PC}_{71} \mathrm{BM}$, we introduced fluorine atoms ${ }^{30}$ to the end-capped acceptor units and obtained two new acceptor units, namely, 4-fluoro-1H-indene-1,3(2H)-dione and 4,7-difluoro$1 \mathrm{H}$-indene-1,3(2H)-dione. Various studies have discussed fluorination effects on photovoltaic performance but most focused on modifying molecular internal part to stabilize molecular conformation ${ }^{31,32}$. In addition, the positions of alkyl chains were designed to prevent backbone torsion to highest extent. The different alkyl chains attached to TBDT units for BTID-0F, BTID-1F and BTID-2F were used to ensure their sufficient solubility. The synthesis routes of the three molecules are shown in Supplementary Fig. 2. The dielectric constants of BTID-0F, BTID-1F and BTID-2F are calculated as ca. 4.0 (Supplementary Table 1, Supplementary Fig. 3a), which was a high value among the conjugated small molecules or polymers ${ }^{33}$.

Absorption spectra of solution and films are shown in Fig. $1 \mathrm{~b}$. The absorption coefficient in solution of the three molecules denoted the competition between the content of alkyl chains and fluorine atoms. Although, four fluorine atoms were introduced into BTID-2F, the alkyl chain (no contribution to absorption) content of which was the highest. Thus, BTID-2F exhibited the lowest absorption coefficient in solution, whereas BTID-1F showed the highest. Furthermore, introduction of fluorine not only enhanced absorption coefficient gradually but also slightly redshifted the absorption spectrum. From solution to films, all the molecules clearly manifested $c a .70 \mathrm{~nm}$ redshifts, illustrating good aggregation in films. With the increase of fluorine atoms, the intensity ratios of the shoulder peak (attributed to $\pi-\pi$ stacking) to the absorption peak from internal charge transfer increased. The different tendencies of film absorption coefficient and the solution absorption coefficient further revealed that fluorination led to dense and ordered $\pi-\pi$ stacking.

Ultraviolet photoelectron spectroscopy (UPS) was carried out to obtain $\mathrm{IP}_{\mathrm{D}}$ in pristine films and blends with $\mathrm{PC}_{71} \mathrm{BM}$ on PEDOT:PSS/ITO substrates (Fig. 1c) ${ }^{34}$, and the IP and EA (electron affinity) of $\mathrm{PC}_{71} \mathrm{BM}$ were obtained from literatures ${ }^{35}$. The $\mathrm{IP}_{\mathrm{D}}$ of the three pristine films were $4.91,4.98$ and $5.05 \mathrm{eV}$, respectively, indicating fluorination of the end acceptors increase $\mathrm{IP}_{\mathrm{D}}$ comparing with those of fluorine atoms attached to the internal part of polymers ${ }^{36,37}$. The detailed $-\mathrm{IP}_{\mathrm{D}}$ and $-\mathrm{EA}_{\mathrm{D}}$ of the three molecules are shown Fig. 1d, and the $E A_{D}$ was calculated from: $\mathrm{EA}_{\mathrm{D}}=-\left(E_{\mathrm{g}}^{\mathrm{opt}}-\mathrm{IP}_{\mathrm{D}}\right)\left(E_{\mathrm{g}}^{\mathrm{opt}}\right.$ is optical energy bandgap). However, after blending with $\mathrm{PC}_{71} \mathrm{BM}$, the $\mathrm{IP}_{\mathrm{D}}$ were changed to $4.98,4.92$ and $4.95 \mathrm{eV}$ for BTID-0F, BTID-1F and BTID-2F, and their corresponding changed values were 0.09 , -0.05 and $-0.09 \mathrm{eV}$. The change from pristine to blended films was mainly due to the shifts in work function (Fig. 1c) indicating dipole polarity differs after $\mathrm{PC}_{71} \mathrm{BM}$ addition. Changes in dipole direction after fluorination were probably due to various surface enrichment and vertical distributions, which will be discussed in the later section. Moreover, the molecules based on the new designed $\pi$-bridge exhibited low highest occupied molecular 
a
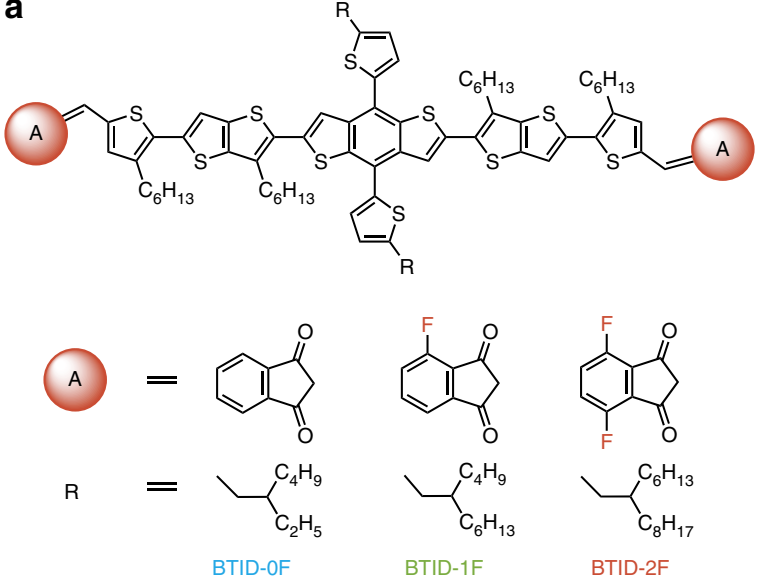

C

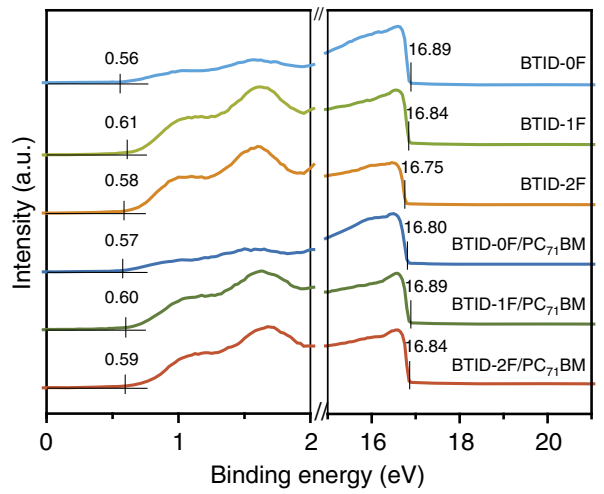

b

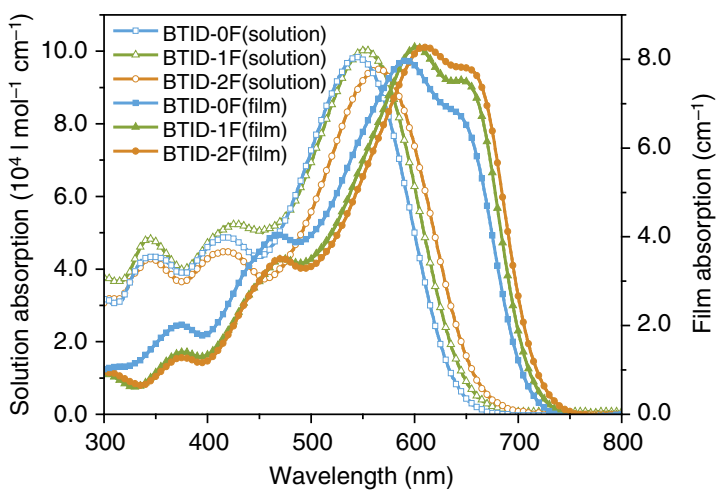

d

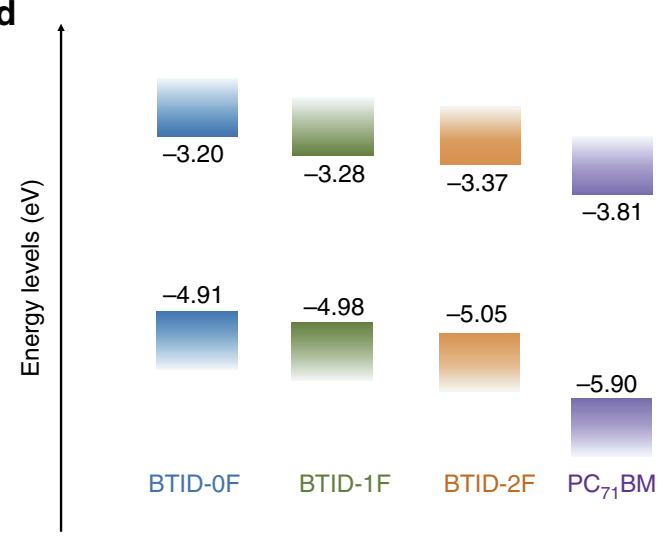

Figure 1 | Molecular structures and properties. (a) Small molecular structures; (b) solution coefficient of samples in chloroform (left $Y$ axis) and film (right $Y$ axis); (c) UPS results of the pristine films and blended films with $\mathrm{PC}_{71} \mathrm{BM}$; and (d) molecular energy levels measured from UPS, the lowest unoccupied molecular orbital (LUMO) levels calculated from optical bandgap and UPS.

orbital (HOMO) levels (measured by cyclic voltammetry, Supplementary Fig. 3b,c), which is the basis for obtaining high $V_{\text {oc }}$ in $\mathrm{BHJ}$ OSCs ${ }^{5}$.

To check the miscibility and molecular surface free energies, the contact angles of chloroform solution of three materials were measured on $\mathrm{ZnO} / \mathrm{ITO}$ and PEDOT/ITO substrates (Supplementary Fig. 3d,e). The increase of contact angles for the fluorinated molecules indicated fluorination lowered the surface free energies of small molecules. On the other hand, the increase of deviation in contact angles between fluorinated molecules and $\mathrm{PC}_{71} \mathrm{BM}$ indicated fluorination also reduced the miscibility of small molecules with $\mathrm{PC}_{71} \mathrm{BM}$. These results implied that surface free energies and miscibility were successfully adjusted through molecular design.

Fabrication and performance of inverted solar cells. To investigate the photovoltaic properties of the three small molecules, devices with a structure of ITO/ZnO/active layer/ $/ \mathrm{MoO}_{x} / \mathrm{Ag}$ were fabricated (Fig. 2a). The highest PCE for BTID-0F, BTID-1F and BTID-2F were 8.30, 10.4 and $11.3 \%$, and other detailed parameters are shown in Fig. 2b,c and Table 1, and the optimization process of $D: A$ ratios is shown in Supplementary Table 2. The device based on BTID-2F was certified at an accredited laboratory, certifying a PCE of $11.0 \%$ (Supplementary Fig. 4a-e). Notably, the active layers of all devices were obtained without any additives and post-treatment, which were facilitated for future industrial manufacturing ${ }^{38}$.

The high efficiencies of the three materials were ascribed to simultaneous increment in $V_{\mathrm{oc}}, J_{\mathrm{sc}}$ and FF. The high $V_{\mathrm{oc}}$ was in good agreement with the $\mathrm{IP}_{\mathrm{D}}$ of their blends obtained by UPS. As calculated from the formula: $e V_{\text {oc }}=\mathrm{IP}_{\mathrm{D}}-\mathrm{EA}_{\mathrm{A}}-\Delta V$ (refs 39,40), the $V_{\mathrm{oc}}$ losses $(\Delta V)$ based on all three small molecules were $c a .0 .2 \mathrm{eV}\left(-\mathrm{IP}_{\mathrm{D}}\right.$ and $-\mathrm{EA}_{\mathrm{A}}$ values shown in Fig. 1d), which were much less than empirical value $0.3-0.5 \mathrm{eV}$ reported ${ }^{39,40}$. The low $V_{\text {oc }}$ loss were attributed to their high dielectric constant (Supplementary Fig. 3a, Supplementary Table 1$)^{33,40}$ and ideal morphologies, which will be discussed in following sections. The $J_{\mathrm{sc}}$ of BTID-2F and BTID-1F cells was higher than that of BTID-0F cells partly because of the larger photocurrent generated in the red region (Fig. 2c). On the other hand, the enhancements of FF and partial $J_{\text {sc }}$ for BTID-2F and BTID-1F compared with BTID-0F, illustrated lower loss of FF and $J_{\mathrm{sc}}$ with fluorination. This phenomenon could be supported by the relation of photocurrent density $\left(J_{\mathrm{ph}}\right)$ versus effective voltage $\left(V_{\text {eff }}\right)$ (Fig. $\left.2 \mathrm{~d}\right)$, where $J_{\mathrm{ph}}=J_{\mathrm{L}}-J_{\mathrm{D}}\left(J_{\mathrm{L}}\right.$ and $J_{\mathrm{D}}$ are the current density under illumination and in the dark) and $V_{\text {eff }}=V_{0}-V_{\mathrm{a}}, \quad\left(V_{0}\right.$ is the voltage at $J_{\mathrm{ph}}=0$ and $V_{\mathrm{a}}$ is the measured voltage under different current density). The ratios of $J_{\mathrm{ph}} / J_{\mathrm{ph}, \mathrm{sat}}$ are used to judge the overall efficiency of exciton dissociation and charge collection $^{2,41}$. Under short-circuit condition, the ratios were $0.93,0.95$ and 0.96 for BTID-0F, BTID-1F and BTID-2F, suggesting the effective exciton dissociation of the three molecules, especially for BTID-1F and BTID-2F. Under maximal power output circumstances $\left(V_{\text {eff }}=0.2 \mathrm{~V}\right)$, the ratios of $J_{\mathrm{ph}} / J_{\mathrm{ph}, \text { sat }}$ were $0.71,0.83$ and 0.86 , respectively, indicating a considerably higher charge collection and lower bimolecular recombination after fluorine substitution. The superior $J_{\mathrm{ph}}-V_{\text {eff }}$ characteristics clearly demonstrated that 
a

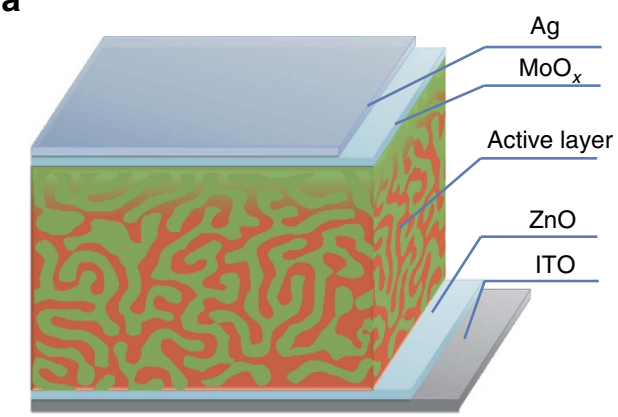

C

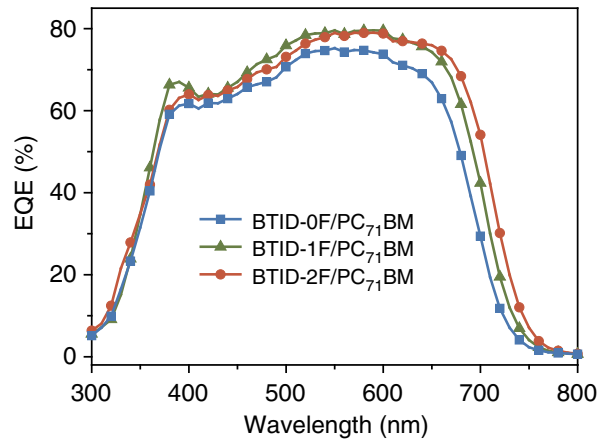

b

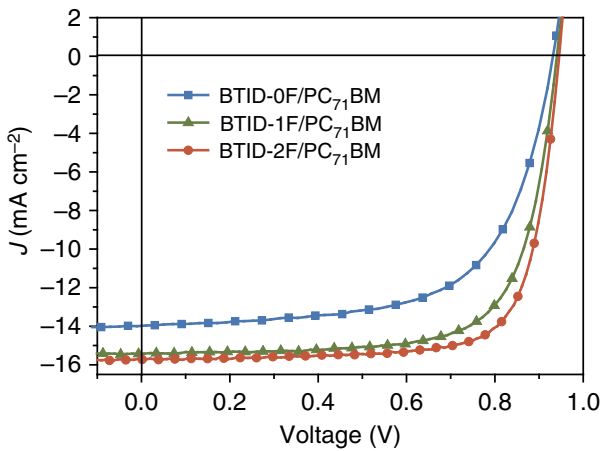

d

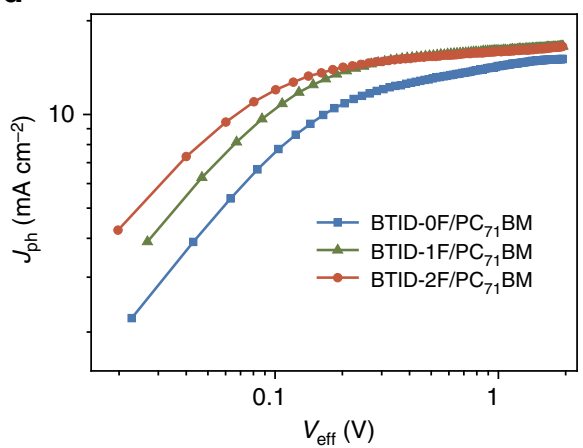

Figure 2 | Device structures and photovoltaic properties for inverted solar cells. (a) Structures of inverted device; (b) optimized $J-V$ curves for inverted devices; (c) EQE corresponding to devices in $\mathbf{b}, \mathbf{d}$ photocurrent density versus effective voltage $\left(J_{\mathrm{ph}}-V_{\text {eff }}\right)$ characteristics for devices under constant incident light intensity (AM 1.5 G, $100 \mathrm{~mW} \mathrm{~cm}^{-2}$ ).

\begin{tabular}{|c|c|c|c|c|c|}
\hline \multirow[t]{2}{*}{ Donors } & \multirow[t]{2}{*}{$V_{\text {oc }}(\mathrm{V})$} & \multirow[t]{2}{*}{ 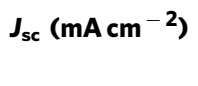 } & \multirow[t]{2}{*}{ FF (\%) } & \multicolumn{2}{|c|}{ PCE (\%) } \\
\hline & & & & Best & Average \\
\hline BTID-OF & 0.93 & 14.0 & 64.0 & 8.30 & 8.21 \\
\hline BTID-1F & 0.94 & 15.3 & 72.0 & 10.4 & 10.37 \\
\hline BTID-2F & 0.95 & 15.7 & 76.0 & 11.3 & 11.08 \\
\hline
\end{tabular}

fluorination could reduce bimolecular recombination, thereby improving $J_{\mathrm{sc}}$ and FF simultaneously.

Hierarchical morphology. The small loss in $J_{\mathrm{sc}}, \mathrm{FF}$ and $V_{\mathrm{oc}}$ for devices based on the three molecules and their variation in loss should be ascribed to the optimized but different lateral/vertical phase distributions. To study morphologies in the lateral direction, we characterized small molecules/ $\mathrm{PC}_{71} \mathrm{BM}$ blending films on $\mathrm{ZnO} / \mathrm{Si}$ substrates and pristine films on $\mathrm{Si}$ substrates by grazing incidence wide-angle X-ray scattering (GIWAXS) (Fig. 3a-f), and their corresponding one-dimension curves are shown in Fig. $3 g$,h. Whether in pristine or blended films, all three molecules exhibited preferable edge-on molecular packing orientation with a small ratio of face-on packing orientation, because evident multiple higher-order $(h 00)$ reflections in the out-of-plane direction and an evident (010) reflection of $\pi-\pi$ stacking in the in-plane direction were observed for all samples. From the calculated faceon to edge-on ratios (Supplementary Table 3), it could be easily found: in pristine films, molecules adopted a more favourable edge-on packing mode; while in blends, the ratios of face-on to edge-on orientation were similar for the three molecules.
The differences in $d$-spacing in the (100) direction were ascribed to the varying lengths of the alkyl (corresponds to the short axis periodicity). The $\pi-\pi$ stacking distance of BTID-0F, BTID-1F and BTID-2F were $3.63,3.57$ and $3.55 \AA$, illustrating a more condensed stacking in the $\pi-\pi$ direction after fluorination; a result was consistent with the absorption in films. The coherence lengths calculated from the $\pi-\pi$ stacking (010) peaks were 51.0, 67.3 and 70.4 $\AA$ for BTID-0F, BTID-1F and BTID-2F, suggesting fluorination of end-capped units increases order range. In comparison with pristine film (Fig. $3 \mathrm{a}-\mathrm{c}$ ), additions of $\mathrm{PC}_{71} \mathrm{BM}$ decreased the coherence length by $13.2,10.4$ and $2 \%$ for BTID0F, BTID-1F and BTID-2F, indicating that fluorination decreases the influence of $\mathrm{PC}_{71} \mathrm{BM}$ on molecular aggregation, especially for BTID-2F; the phenomenon could be ascribed to their decreased miscibility with $\mathrm{PC}_{71} \mathrm{BM}$. In addition, $\mathrm{PC}_{71} \mathrm{BM}$ in all blends exhibited strong aggregation with coherence lengths $c a$. $20 \AA$ (Supplementary Table 3). The good aggregations for $\mathrm{PC}_{71} \mathrm{BM}$ and small molecules reduced interfacial energy disorder, which was beneficial for further lowering loss of $V_{\text {oc }}$ (ref. 22).

The morphologies were further investigated by atomic force spectroscopy (AFM) and transmission electron microscopy (TEM). The domain in AFM phase images (Fig. 4a-c) increased in size after fluorinations in accordance with their enhanced crystalline. As seen in TEM images (Fig. $4 \mathrm{~d}-\mathrm{f}$ ), nanostructures were observed for BTID-0F/PC ${ }_{71} \mathrm{BM}$ blends with diameters $c a$. $25 \mathrm{~nm}$. After fluorination, the diameters of nanostructure increased evidently for BTID-1F/PC ${ }_{71} \mathrm{BM}$ blends because of increased order range. Interestingly, with a further increase in fluorine atoms, an evident network of whiskers with diameter $c a$. $15 \mathrm{~nm}$ was observed, interpenetrating in the larger domains.

Resonant soft X-ray scattering (RSoXS) was employed to investigate the above mentioned fine microstructure (Fig. 4g). With increment of fluorine atoms, the dominated domain size increased from 24 to 43 , and finally to $53 \mathrm{~nm}$, as in good 


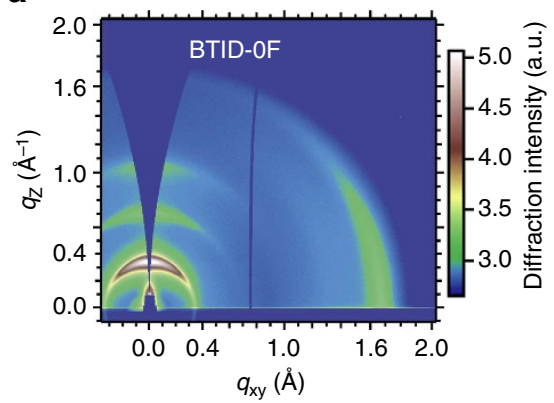

d

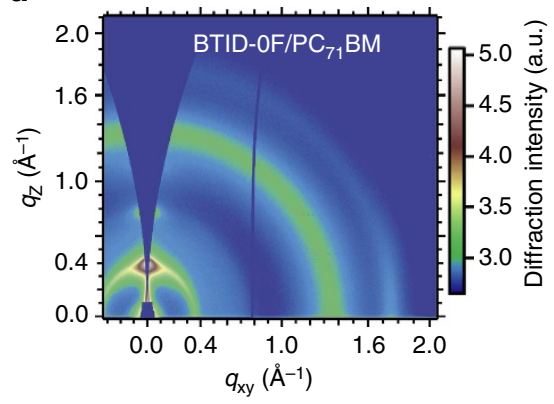

g

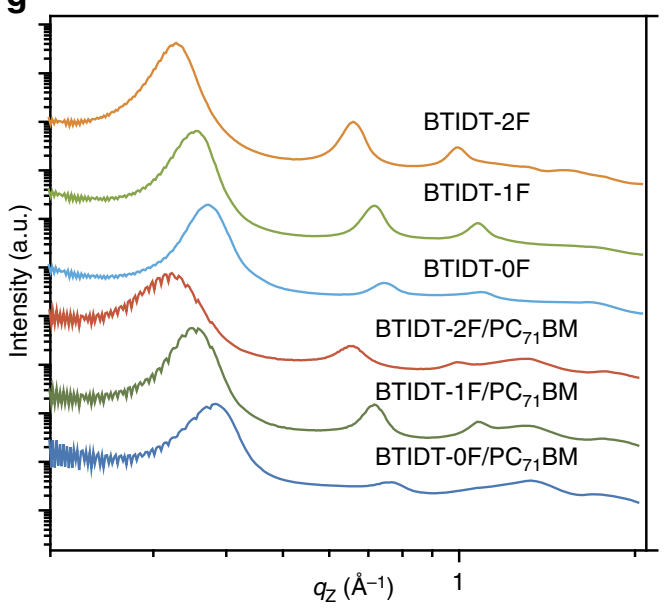

b

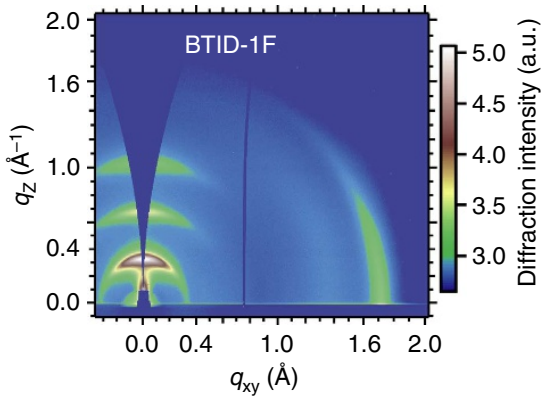

e

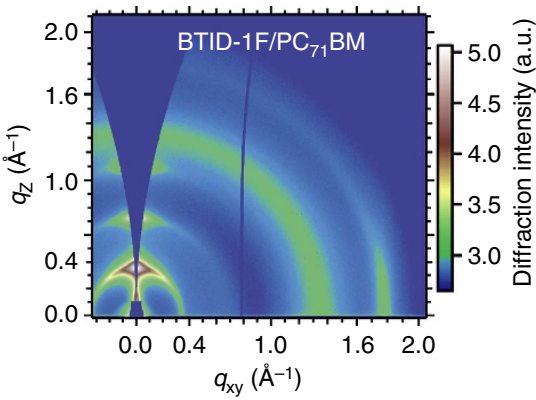

C

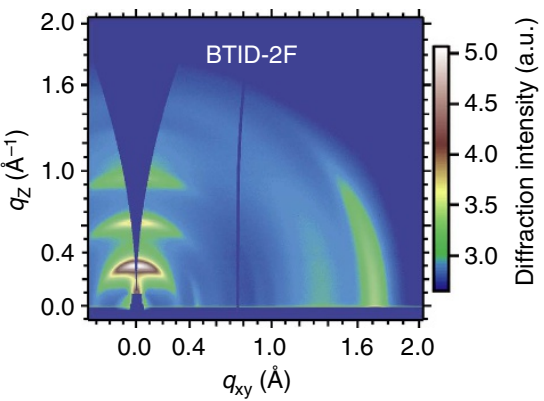

$\mathbf{f}$

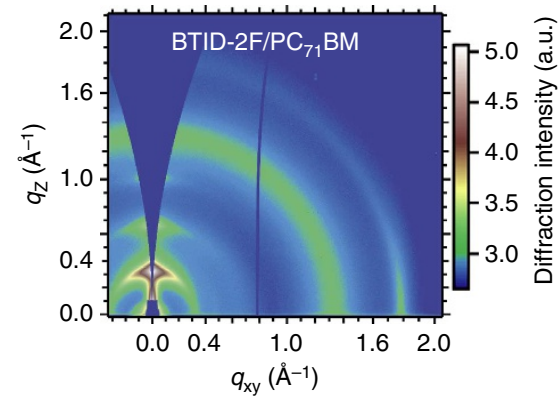

h

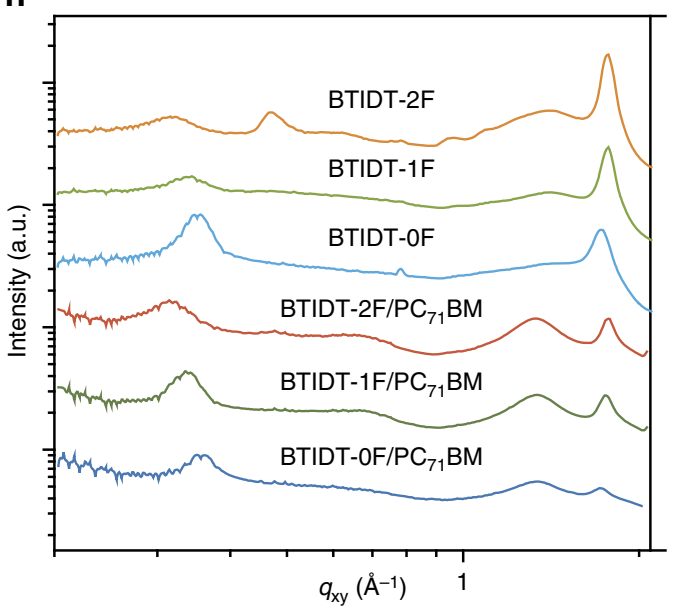

Figure 3 | Microstructures of pristine and blend films. (a-f) GIWAXS images in pristine films on Si substrate and GIWAXS images in blends films on $\mathrm{ZnO} / \mathrm{Si}$ substrates; (g) corresponding out-of-plane curves; and (h) corresponding in-plane curves.

agreement with the increased crystallinity. However, by carefully analysing the RSoXS profiles, we could see that the scattering distribution could be fitted by two log-normal functions, with the other peak in the longer $q$-values: $c a .0 .25 \mathrm{~nm}^{-1}$ for BTID-2F (corresponding to domain size of $12.8 \mathrm{~nm}$, calculated from plot fitting in Supplementary Fig. 5) and a less evident interference appeared at $c a .0 .22 \mathrm{~nm}^{-P}$ for BTID-1F (corresponding to domain size of $14.4 \mathrm{~nm}$ ). As for BTID-0F, no obvious difference was found between the two fitting domain sizes $(24$ and $20 \mathrm{~nm}$ ). In other words, BTID-2F/PC $\mathrm{PC}_{71} \mathrm{BM}$ and BTID-1F/PC ${ }_{71} \mathrm{BM}$ blends demonstrated formation of a hierarchical morphology with secondary domain sizes, and the secondary domain size was closed to the exciton diffusion length of $c a .10 \mathrm{~nm}$ (Fig. 4h). Moreover, the relative domain purity for BTID-0F, BTID-1F and BTID-2F was calculated as $0.70,0.93$ and 1 , in good agreement with increased crystallinity and decreased miscibility by increased fluorine introduction (Fig. 4g,h). The hierarchical morphology is reported to well balance domain size and purity to facilitate charge separation and transfer; the smaller donor phase accounts for charge separation, whereas the larger donor phase is responsible for charge transport ${ }^{42,43}$. Therefore, the hierarchical morphology consisting 10-20 $\mathrm{nm}$ structure and enhanced domain purity could increase $\mathrm{FF}$ and $J_{\mathrm{sc}}$ simultaneously.

Surface enrichment and vertical phase distribution. X-ray photoelectron spectroscopy (XPS) was carried out to study surface enrichment and vertical phase distribution. In the blends of small molecules: $\mathrm{PC}_{71} \mathrm{BM}$, sulfur and fluorine atoms could be used as characteristic elements of the small molecules due to the absence of the two elements of $\mathrm{PC}_{71} \mathrm{BM}$. A parameter named 'surface enrichment degree' was introduced to characterize surface enrichment, which was equal to divide the $\mathrm{S}: \mathrm{C}$ (or F:C) ratio measured through XPS by the ideal $\mathrm{S}: \mathrm{C}$ (or $\mathrm{F}: \mathrm{C}$ ) ratio calculated from D:A ratio (Fig. 5a). The histogram in Fig. 5a illustrated that all three molecules were prone to accumulating on the surface. With fluorination, surface enrichment was enhanced from 1.69 for BTID-0F to 1.79 for BTID-1F and 1.81 for BTID-2F, as 
a

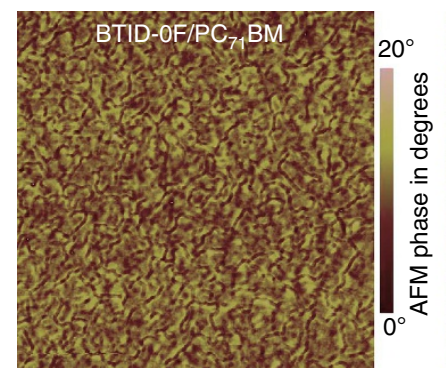

d

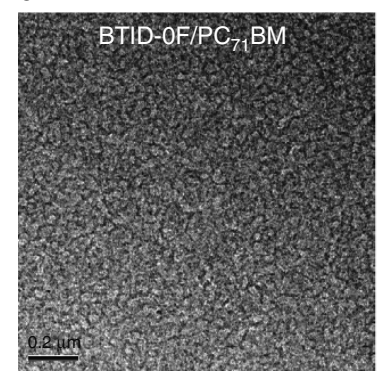

g

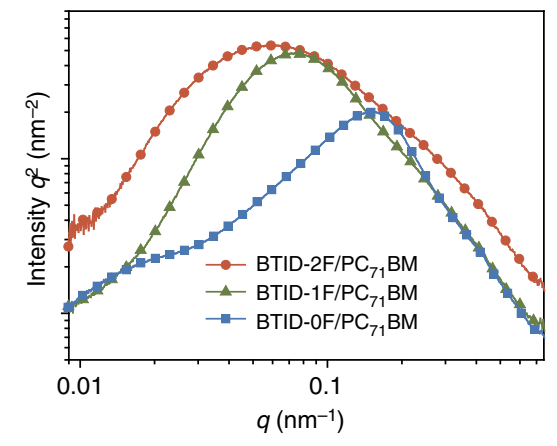

b

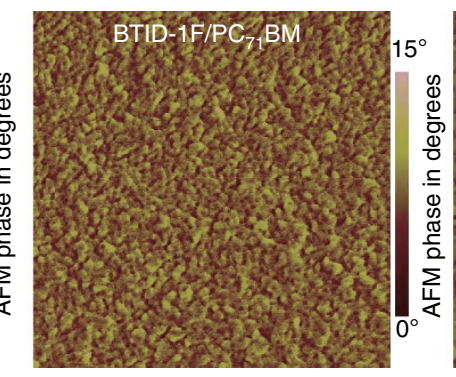

e

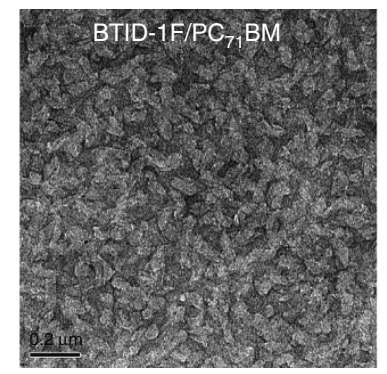

c

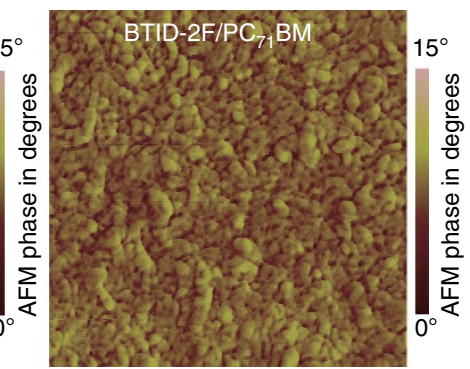

f

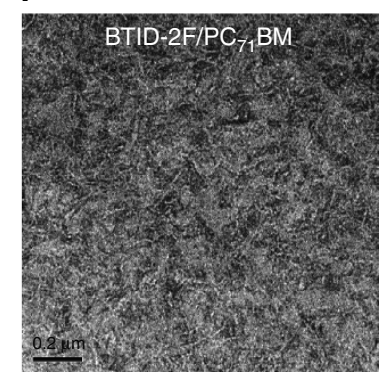

h

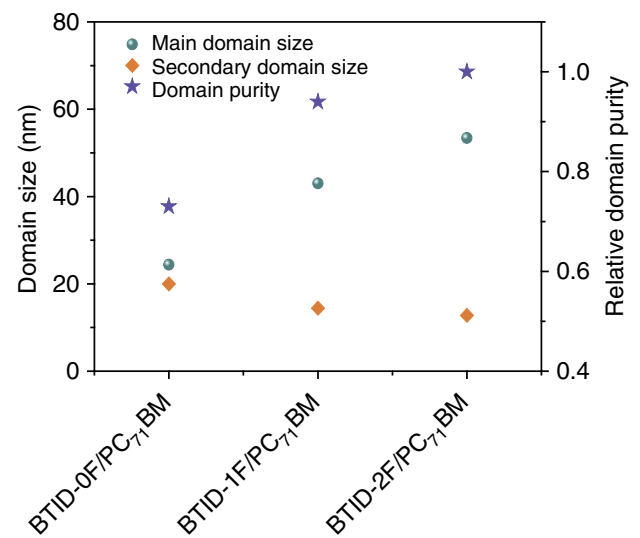

Figure 4 | Lateral morphologies and microstructures of blend films on ZnO/ITO substrates. (a-c) AFM phase images; (d-f) TEM images; (g) RSoXs profiles; and (h) relative domain purity and domain sizes.

calculated from S:C ratios. The 'surface enrichment degree' calculated from F:C ratios were markedly higher (2.1 for BTID$\mathbf{1 F}$ and 2.6 for BTID-2F) than those of S:C ratios, indicating that the fluorine-substituted groups are more prone to be enriched at the active layer surface.

Subsequently, in-depth XPS measurements in the vertical direction of BTID-0F and BTID-2F blends on $\mathrm{ZnO} / \mathrm{ITO}$ substrates were carried out to characterize the vertical phase distribution (Fig. 5b). We defined the active layer/ZnO interface by the appearance of a large amount of $\mathrm{Zn}$ element. The corrosion time of active layer was slightly different because of the material and film thickness differences. BTID-0F exhibited enrichments both on top surface (air/active layer) and bottom interface (active layer/ZnO). As for BTID-2F, surface enrichment degree was increased evidently, whereas enrichment at the bottom and bulk was suppressed. As calculated from F:C ratios, the top surface was nearly $100 \%$ of BTID-2F (Supplementary Methods), indicating an electron blocking layer was formed at the active layer $/ \mathrm{MoO}_{x}$ interface. The more directional vertical phase distribution and formation of electron blocking layer facilitated the charge extraction/collection and recombination suppression, leading to a higher $\mathrm{FF}$ and $J_{\mathrm{sc}}$ in inverted devices than those of conventional devices for BTID-2F (Supplementary Fig. 6,
Supplementary Table 4). Moreover, the vertical distribution and surface enrichment reduced recombination and increased the build-in potential of inverted devices in comparison of its conventional devices, which thereby further reduced $V_{\text {oc }}$ loss. The influence of surface enrichment and vertical phase separation on the device performance was further certified in the Supplementary evidence of the role of surface enrichment and vertical phase separation (Supplementary Discussions, Supplementary Fig. 7, Supplementary Tables 3 and 5).

The surface enrichment and vertical phase distribution were further manifested by results of charge carrier mobility (Fig. 5c). All the carrier mobility were used average values measured by space-charge limited current method (Supplementary methods), obtained from thickness between 100 and $130 \mathrm{~nm}$ (Supplementary Fig. 8). The average hole mobility of pristine film for BTID-0F, BTID-1F and BTID-2F were $8.7 \times 10^{-4}$ $\mathrm{cm}^{2} \mathrm{~V}^{-1} \mathrm{~s}^{-1}, 6.4 \times 10^{-4} \mathrm{~cm}^{2} \mathrm{~V}^{-1} \mathrm{~s}^{-1}$ and $3 \times 10^{-4} \mathrm{~cm}^{2} \mathrm{~V}^{-1}$ $\mathrm{s}^{-1}$, the decrease of hole mobility with fluorination should be mainly resulted from their decreased face-on packing ratios (Supplementary Table. 3) and longer alkyl chain lengths. However, after blending of $\mathrm{PC}_{71} \mathrm{BM}, \mathbf{B T I D - 2 F}$ showed markedly higher hole mobility than BTID-0F, the average of which were $1.4 \times 10^{-3} \mathrm{~cm}^{2} \mathrm{~V}^{-1} \mathrm{~s}^{-1}$ and $4.7 \times 10^{-4} \mathrm{~cm}^{2} \mathrm{~V}^{-1} \mathrm{~s}^{-1}$. On the 
a

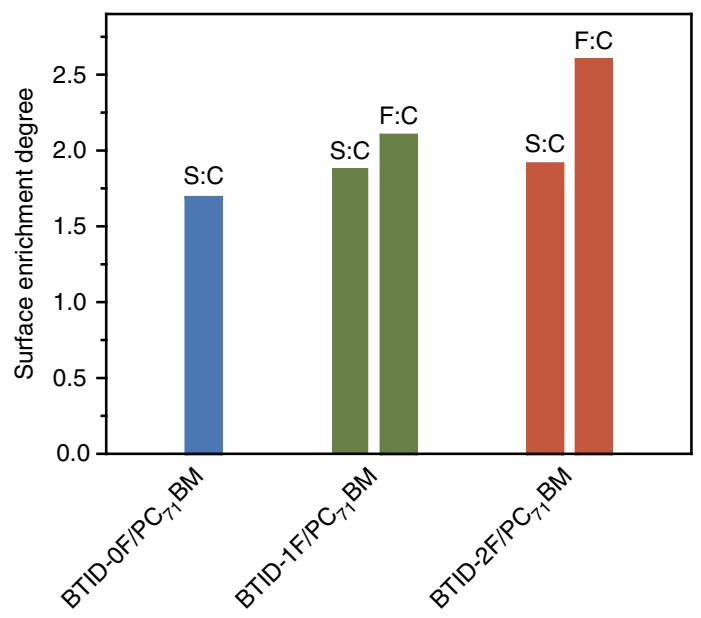

C

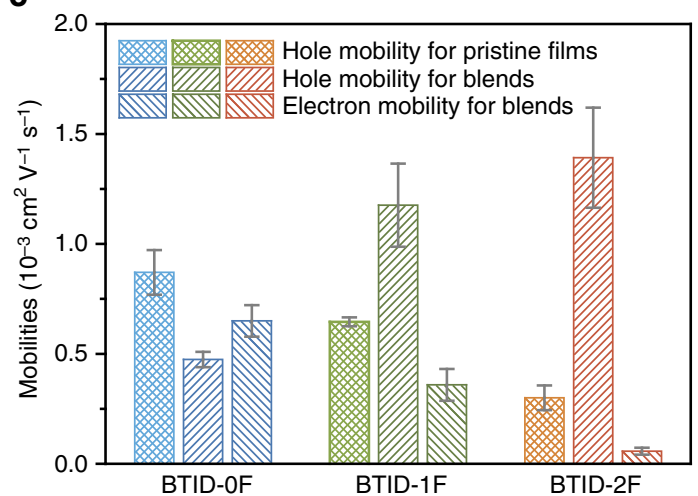

b

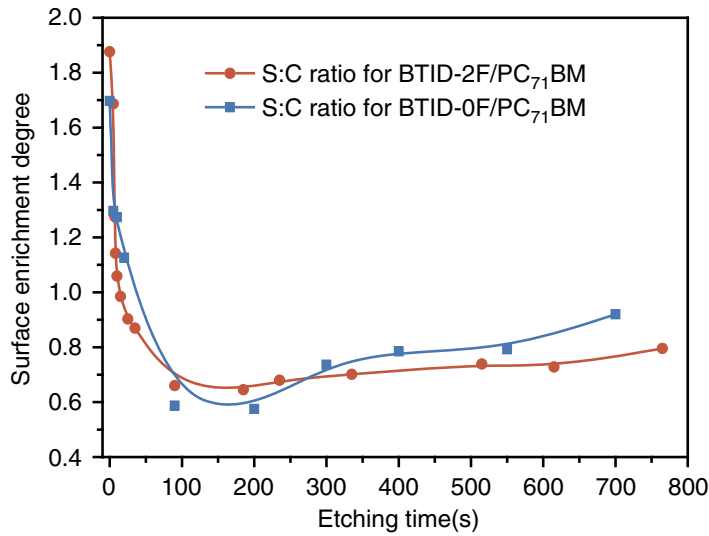

d

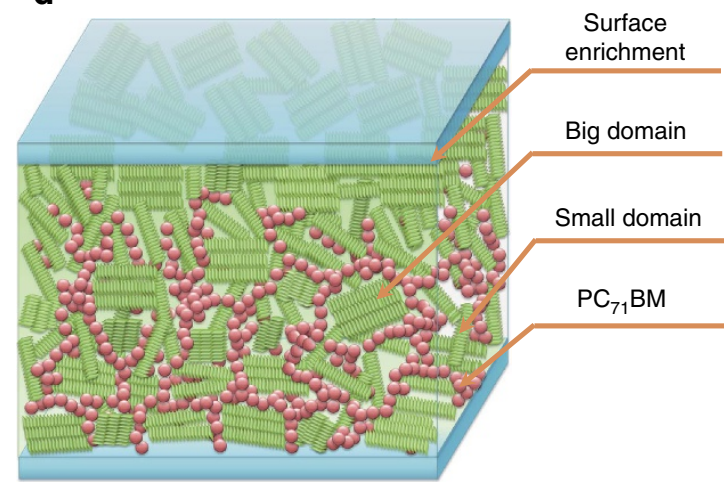

Figure 5 | Vertical morphologies of blended films. (a) Surface enrichments of $B T I D-O F / P C_{71} B M, B T I D-1 F / P C C_{71} B M$ and $B T I D-2 F / P C C_{71} B M$ on $Z n O / I T O$ substrate; (b) in-depth XPS profiles of BTID-OF/PC ${ }_{71} B M$ and BTID-2F/PC ${ }_{71} B M$ on $Z n O / I T O$ substrates, the line were obtained from fitting all the S:C value from various etching time; (c) carrier mobilities of blends and pristine films, the average values were obtained from the mobility measured from the thickness between 100 and $130 \mathrm{~nm}$, the error bars come from the mobility value from thickness differences and their measurements errors; and (d) schematic illustrations of lateral and vertical phase distribution of BTID-2F/PC 71 BM.

other hand, the electron mobility of BTID-2F/PC ${ }_{71} \mathrm{BM}$ blends was considerably lower than that of BTID-0F/PC ${ }_{71} \mathrm{BM}$ blends. The hole and electron mobility of BTID-1F/PC ${ }_{71} B M$ were in between. The different trends for hole and electron mobility could be explained by the largest surface enrichment of BTID-2F, which facilitated hole transport while blocked electron transport. The increase in hole mobility of blends compared with pristine films for fluorine-substituted molecules could be explained by their increased ratios of face-on to edge-on after $\mathrm{PC}_{71} \mathrm{BM}$ blending (Supplementary Table 3) and favourable backbone orientation for vertical charge transport ${ }^{10}$.

\section{Discussion}

Based on hierarchical morphology at lateral direction and vertical phase distribution, the morphology of active layer was schematically shown as Fig. $5 \mathrm{~d}$ for $\mathrm{BTID}-2 \mathrm{~F} / \mathrm{PC}_{71} \mathrm{BM}$. In lateral direction, BTID-2F formed a hierarchical morphology with high domain purity, composing domains with diameters of $c a .53 .0 \mathrm{~nm}$ and an interpenetrating whisker network with diameters of $c a$. $12.8 \mathrm{~nm}$. A similar hierarchical morphology for BTID-1F/ $\mathrm{PC}_{71} \mathrm{BM}$ was observed but with a lower domain purity, while no obvious difference for two domain sizes in BTID-0F/PC ${ }_{71} \mathrm{BM}$. In vertical direction, BTID-2F formed hole-transporting layers on the top interface and more directional vertical phase distributions of the active layer than those of BTID-0F.
Therefore, fluorination-enabled optimal hierarchical morphology and surface enrichment, which could increase $V_{\mathrm{oc}}, J_{\mathrm{sc}}$ and FF simultaneously and thereby obtained a high PCE in inverted devices. To further verify the influence of hierarchical morphology on device performance (Supplementary Discussions), the substrate temperature was increased from $28^{\circ} \mathrm{C}$ (normal) to $40^{\circ} \mathrm{C}$ (hot) during film formation. Due to a faster solvent evaporation on hot substrate, the hierarchical morphology was not formed as proved by TEM images and RSoXS images (Supplementary Fig. 9). As a consequence, the device performance based on the hot substrate was decreased (Supplementary Table 6). Hence, the hierarchical morphology is one of the most important factors to obtain high performance devices.

For the molecular design, acceptor-donor-acceptor have been widely used for organic photovoltaic. To obtain a low HOMO level and a high hole mobility simultaneously, a novel $\pi$-bridge between donor and acceptor unit, 2-(thiophen-2-yl) thieno $[3,2-b]$ thiophene was introduced, which presented an inner gradient-decreased electron density distribution, and facilitated the backbone charge transfer. Different from that of polymers, the end acceptor played an important role to tune molecular packing and miscibility with $\mathrm{PC}_{71} \mathrm{BM}$. Therefore, fluorinated end-capped acceptor were introduced in the molecular design, which lowered surface tension and their miscibility with PCBM, As a result, the lateral and vertical morphology of the active layer was optimized. BTID-2F formed a hierarchical morphology in the active layer, 
which will inspire more investigations on the effects of $\pi$-bridges and end acceptors for high performance OSCs.

In summary, by combining traditional molecular design strategy with fine tuning surface tension and miscibility with $\mathrm{PC}_{71} \mathrm{BM}$ by fluorination, we designed and synthesized three novel molecules, BTID-0F, BTID-1F and BTID-2F, with incremental fluorine atoms. The three molecules exhibited excellent molecular properties, such as low HOMO levels, good crystallinity and high hole mobility, because of well-designed molecular structures, including new gradient-decreased electron density $\pi$-bridges and proper polarity of aromatic acceptors. Through device measurement and morphology characterization, we emphasized the importance of fluorination to hierarchical morphology, surface enrichment and directional vertical phase distribution. The optimal morphology was beneficial to charge transfer, charge collection and recombination suppression, which reduced the loss of $V_{\mathrm{oc}}, J_{\mathrm{sc}}$ and FF simultaneously. As a result, a record PCE of $11.3 \%$ was obtained in inverted OSCs based on small molecules, with $V_{\mathrm{oc}}$ of $0.95 \mathrm{~V}, J_{\mathrm{sc}}$ of $15.7 \mathrm{~mA} \mathrm{~cm}^{-2}$ and $\mathrm{FF}$ of $76 \%$.

\section{Methods}

Solar cell fabrication and measurements. Inverted devices were fabricated with a structure of glass/ITO/ZnO/donor:acceptor/ $\mathrm{MoO}_{x} / \mathrm{Ag}$. The $\mathrm{ZnO}$ precursor solution was prepared by dissolving $0.14 \mathrm{~g}$ of zinc acetate dihydrate $\left(\mathrm{Zn}\left(\mathrm{CH}_{3} \mathrm{COO}\right)_{2} \cdot 2 \mathrm{H}_{2} \mathrm{O}, 99.9 \%\right.$, Aldrich $)$ and $0.5 \mathrm{~g}$ of ethanolamine $\left(\mathrm{NH}_{2} \mathrm{CH}_{2} \mathrm{CH}_{2} \mathrm{OH}, 99.5 \%\right.$, Aldrich) in $5 \mathrm{ml}$ of 2-methoxyethanol $\left(\mathrm{CH}_{3} \mathrm{OCH}_{2}\right.$ $\mathrm{CH}_{2} \mathrm{OH}, 99.8 \%$, J\&K Scientific). Patterned ITO glass with a sheet resistance of $15 \Omega \mathrm{sq}^{-1}$ was purchased from CSG HOLDING Co., Ltd. The ITO-coated glass substrates were cleaned by ultrasonic treatment in detergent, DI water, acetone and isopropyl alcohol under ultrasonication for $20 \mathrm{~min}$ at each step. A thin layer of $\mathrm{ZnO}$ precursor was spin-coated at 5,000 r.p.m. onto the ITO surface. After being baked at $200^{\circ} \mathrm{C}$ for $30 \mathrm{~min}$, the substrates were transferred into a nitrogen-filled glove box. The mixture of small molecules and $\mathrm{PC}_{71} \mathrm{BM}$ with total concentration ca. $18 \mathrm{mg} \mathrm{ml}^{-1}$ stirred at $60^{\circ} \mathrm{C}$ in chloroform for $\mathrm{ca} .0 .5 \mathrm{~h}$ until they are intensively dissolved. Subsequently, the active layer was spin-coated from chloroform solutions of blends. Finally, a layer of $c a .5 \mathrm{~nm} \mathrm{MoO}$ and then an Ag layer of $c a .160 \mathrm{~nm}$ was evaporated subsequently under high vacuum $\left(<1 \times 10^{-4} \mathrm{~Pa}\right)$.

Conventional devices were fabricated with a structure of glass/ITO/ PEDOT:PSS/Ca/Al. The ITO-coated glass substrates were cleaned by the same procedure with inverted devices. A thin layer of PEDOT:PSS was spin-coated at 4,000 r.p.m. onto the ITO surface. After being baked at $150^{\circ} \mathrm{C}$ for $15 \mathrm{~min}$, the substrates were transferred into a nitrogen-filled glove box. The mixture of small molecules and $\mathrm{PC}_{71} \mathrm{BM}$ with total concentration $\mathrm{ca} .18 \mathrm{mg} \mathrm{ml}^{-1}$ stirred at $60^{\circ} \mathrm{C}$ in chloroform for $c a$. $0.5 \mathrm{~h}$ until they intensively dissolved. Subsequently, the active layer was spin-coated from blend chloroform solutions of small molecules and $\mathrm{PC}_{71} \mathrm{BM}$. Finally, a layer of $\sim 20 \mathrm{~nm} \mathrm{Ca}$ and then $100 \mathrm{~nm}$ Al layer was evaporated under high vacuum $\left(<1 \times 10^{-4} \mathrm{~Pa}\right)$.

Device $J-V$ characteristics was measured under AM $1.5 \mathrm{G}\left(100 \mathrm{~mW} \mathrm{~cm}^{-2}\right)$ using a Newport Thermal Oriel 91159A solar simulator. Light intensity is calibrated with a Newport Oriel PN $91150 \mathrm{~V}$ Si-based solar cell. $J-V$ characteristics were recorded using a Keithley 2400 source-measure unit. Typical cells have device areas of approximately $4 \mathrm{~mm}^{2}$. A mask with well-defined area was used to measure the $J-V$ characteristics as well. EQEs were performed in air with an Oriel Newport system (Model 66902) equipped with a standard Si diode. Monochromatic light was generated from a Newport $300 \mathrm{~W}$ lamp source. We have used mask for BTID-2F, the errors are in $5 \%$.

Supplementary Methods including: characterization methods: (1) molecular structure characterization and calculation (nuclear magnetic resonance, mass spectrometry spectra, discrete Fourier transform); (2) molecular properties characterization (dielectric constant, UPS, ultraviolet-vis spectra, CV, UPS, contact angle); (3) TEM, AFM, XPS and in-depth XPS characterization; (4) GIWAXS characterization; (5) RSoXs characterization; and (6) $J_{\mathrm{ph}}$ and mobility measurements. Calculation methods: (1) calculations of ionization potential of donor $\left(\mathrm{IP}_{\mathrm{D}}\right.$ ) from ultraviolet photoelectron spectroscopy (UPS); (2) calculation of the coherence length $\left(L_{\mathrm{c}}\right)$ of $\mathrm{PC}_{71} \mathrm{BM}$ and small molecules; (3) calculation of domain size and purity from RSoXs ; (4) calculation of surface enrichment degrees; (5) calculation of surface $D: A$ ratio; and (6) calculations of mobility measured from space-charge limited current. Synthesis methods including: materials and synthesis.

Data availability. All relevant data are available from the authors.

\section{References}

1. He, Z. et al. Enhanced power-conversion efficiency in polymer solar cells using an inverted device structure. Nat. Photonics 6, 591-595 (2012).
2. Zhang, S., Ye, L. \& Hou, J. Breaking the $10 \%$ efficiency barrier in organic photovoltaics: morphology and device optimization of well-known PBDTTT polymers. Adv. Energy Mater. 1502529-1502549 (2016).

3. Vohra, V. et al. Efficient inverted polymer solar cells employing favourable molecular orientation. Nat. Photonics 9, 403-408 (2015).

4. Zheng, Z. et al. Over $11 \%$ efficiency in tandem polymer solar cells featured by a low-band-gap polymer with fine-tuned properties. Adv. Mater. 28, 5133-5138 (2016).

5. Zhao, J. et al. Efficient organic solar cells processed from hydrocarbon solvents. Nat. Energy 1, 15027 (2016).

6. Sun, Y. et al. Solution-processed small-molecule solar cells with $6.7 \%$ efficiency. Nat. Mater. 11, 44-48 (2012).

7. Zhang, Q. et al. Small-molecule solar cells with efficiency over $9 \%$. Nat. Photonics 9, 35-41 (2015).

8. Sun, K. et al. A molecular nematic liquid crystalline material for highperformance organic photovoltaics. Nat. Commun. 6, 1-9 (2015).

9. Zhang, J. et al. Solution-processable star-shaped molecules with triphenylamine core and dicyanovinyl endgroups for organic solar cells. Chem. Mater. 23, 817-822 (2010).

10. Deng, D. et al. Effects of shortened alkyl chains on solution-processable small molecules with oxo-alkylated nitrile end-capped acceptors for highperformance organic solar cells. Adv. Energy Mater. 4, 1408538-1408545 (2014).

11. Lin, Y., Li, Y. \& Zhan, X. Small molecule semiconductors for high-efficiency organic photovoltaics. Chem. Soc. Rev. 41, 4245-4272 (2012).

12. Kan, B. et al. A series of simple oligomer-like small molecules based on oligothiophenes for solution-processed solar cells with high efficiency. J. Am. Chem. Soc. 137, 3886-3893 (2015).

13. Wang, Z. et al. Solution-processable small molecules for high-performance organic solar cells with rigidly fluorinated $2,2^{\prime}$-bithiophene central cores. ACS Appl. Mater. Interfaces 8, 11639-11648 (2016).

14. Min, J. et al. A combination of $\mathrm{Al}$-doped $\mathrm{ZnO}$ and a conjugated polyelectrolyte interlayer for small molecule solution-processed solar cells with an inverted structure. J. Mater. Chem. A 1, 11306-11311 (2013).

15. Gautrot, J. E., Hodge, P., Cupertino, D. \& Helliwell, M. 2,6-Diaryl-9,10anthraquinones as models for electron-accepting polymers. New J. Chem. 31, 1585-1593 (2007).

16. Kyaw, A. K. K. et al. Efficient solution-processed small-molecule solar cells with inverted structure. Adv. Mater. 25, 2397-2402 (2013).

17. Long, G. et al. Enhancement of performance and mechanism studies of allsolution processed small-molecule based solar cells with an inverted structure. ACS Appl. Mater. Interfaces 7, 21245-21253 (2015).

18. Cheng, Y.-J., Yang, S.-H. \& Hsu, C.-S. Synthesis of conjugated polymers for organic solar cell applications. Chem. Rev. 109, 5868-5923 (2009).

19. Zhou, H., Yang, L. \& You, W. Rational design of high performance conjugated polymers for organic solar cells. Macromolecules 45, 607-632 (2012).

20. Li, Y. Molecular design of photovoltaic materials for polymer solar cells: toward suitable electronic energy levels and broad absorption. Acc. Chem. Res. 45, 723-733 (2012).

21. He, Z. et al. Single-junction polymer solar cells with high efficiency and photovoltage. Nat. Photonics 9, 174-179 (2015).

22. Elumalai, N. K. \& Uddin, A. Open circuit voltage of organic solar cells: an indepth review. Energy Environ. Sci. 9, 391-410 (2016).

23. Fang, L. et al. Side-chain engineering of isoindigo-containing conjugated polymers using polystyrene for high-performance bulk heterojunction solar cells. Chem. Mater. 25, 4874-4880 (2013).

24. Guo, X. et al. Polymer solar cells with enhanced fill factors. Nat. Photon. 7, 825-833 (2013).

25. Collins, B. A. et al. Absolute measurement of domain composition and nanoscale size distribution explains performance in $\mathrm{PTB}_{7}: \mathrm{PC}_{71} \mathrm{BM}$ solar cells. Adv. Energy Mater. 3, 65-74 (2013).

26. Matsumoto, F. et al. Controlling the polarity of fullerene derivatives to optimize nanomorphology in blend films. ACS Appl. Mater. Interfaces 8, 4803-4810 (2016).

27. Nilsson, S., Bernasik, A., Budkowski, A. \& Moons, E. Morphology and phase segregation of spin-casted films of polyfluorene/PCBM blends. Macromolecules 40, 8291-8301 (2007).

28. $\mathrm{Xu}, \mathrm{Z}$. et al. Vertical phase separation in poly(3-hexylthiophene): fullerene derivative blends and its advantage for inverted structure solar cells. Adv. Funct. Mater. 19, 1227-1234 (2009).

29. Huo, L. et al. Replacing alkoxy groups with alkylthienyl groups: a feasible approach to improve the properties of photovoltaic polymers. Angew. Chem. Int. Ed. 123, 9871-9876 (2011).

30. Kim, J. S. et al. High-efficiency organic solar cells based on end-functionalgroup-modified poly(3-hexylthiophene). Adv. Mater. 22, 1355-1360 (2010).

31. Stuart, A. C. et al. Fluorine substituents reduce charge recombination and drive structure and morphology development in polymer solar cells. J. Am. Chem. Soc. 135, 1806-1815 (2013). 
32. Takacs, C. J. et al. Solar cell efficiency, self-assembly, and dipole-dipole interactions of isomorphic narrow-band-gap molecules. J. Am. Chem. Soc. 134, 16597-16606 (2012).

33. Torabi, S. et al. Strategy for enhancing the dielectric constant of organic semiconductors without sacrificing charge carrier mobility and solubility. Adv. Funct. Mater. 25, 150-157 (2015).

34. Widmer, J., Tietze, M., Leo, K. \& Riede, M. Open-circuit voltage and effective gap of organic solar cells. Adv. Funct. Mater. 23, 5814-5821 (2013).

35. Yoshida, H. Low-energy inverse photoemission study on the electron affinities of fullerene derivatives for organic photovoltaic cells. J. Phys. Chem. C 118, 24377-24382 (2014).

36. Zhang, M., Guo, X., Zhang, S. \& Hou, J. Synergistic effect of fluorination on molecular energy level modulation in highly efficient photovoltaic polymers. Adv. Mater. 26, 1118-1123 (2014).

37. Chen, H.-Y. et al. Polymer solar cells with enhanced open-circuit voltage and efficiency. Nat. Photonics 3, 649-653 (2009).

38. Cui, C. et al. High-performance organic solar cells based on a small molecule with alkylthio-thienyl-conjugated side chains without extra treatments. Adv. Mater. 27, 7469-7475 (2015).

39. Scharber, M. C. et al. Design rules for donors in bulk-heterojunction solar cells towards 10\% energy conversion efficiency. Adv. Mater. 18, 789-794 (2006).

40. Chen, S., Tsang, S.-W., Lai, T.-H., Reynolds, J. R. \& So, F. Dielectric effect on the photovoltage loss in organic photovoltaic cells. Adv. Mater. 26, 6125-6131 (2014).

41. He, Z. et al. Simultaneous enhancement of open-circuit voltage, short-circuit current density, and fill factor in polymer solar cells. Adv. Mater. 23, 4636-4643 (2011).

42. Chen, W. et al. Hierarchical nanomorphologies promote exciton dissociation in polymer/fullerene bulk heterojunction solar cells. Nano Lett. 11, 3707-3713 (2011).

43. Guo, X. et al. Enhanced photovoltaic performance by modulating surface composition in bulk heterojunction polymer solar cells based on PBDTTTC-T/PC ${ }_{71}$ BM. Adv. Mater. 26, 4043-4049 (2014).

\section{Acknowledgements}

We acknowledge the financial support by the Ministry of Science and Technology of China (No 2016YFA0200700), the National Natural Science Foundation of China (Grant Nos 21534003, 91427302, and 21504066) and the 'Strategic Priority Research Program' of the Chinese Academy of Sciences (Grant No XDA09040200). X-ray data was acquired at beamlines 7.3.3 and 11.0.1.2 at the Advanced Light Source, which is supported by the Director, Office of Science, Office of Basic Energy Sciences, of the U.S. Department of Energy under Contract No DE-AC02-05CH11231.

\section{Author contributions}

D.D. designed, synthesized and characterized materials and fabricated devices based on BTID-2F. Y.Z. performed device fabrication of BTID-0F and BTID-1F. B.X. helped device fabrication. J.Z. performed GIWAXS data analysis. Z.W. and W.M. performed GIWAXS/RSoXs measurements and data analysis. L.Z. and Z.W. performed theoretical simulation. J.F. performed dielectric constant measurements. D.D., K.L. and Z.W. prepared manuscript. All authors discussed and commented on the paper. K.L. and Z.W. supervise the project.

\section{Additional information}

Supplementary Information accompanies this paper at http://www.nature.com/ naturecommunications

Competing financial interests: The authors declare no competing financial interests.

Reprints and permission information is available online at http://npg.nature.com/ reprintsandpermissions/

How to cite this article: Deng, D. et al. Fluorination-enabled optimal morphology leads to over $11 \%$ efficiency for inverted small-molecule organic solar cells. Nat. Commun. 7, 13740 doi: $10.1038 /$ ncomms13740 (2016).

Publisher's note: Springer Nature remains neutral with regard to jurisdictional claims in published maps and institutional affiliations.

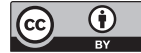

This work is licensed under a Creative Commons Attribution 4.0 International License. The images or other third party material in this article are included in the article's Creative Commons license, unless indicated otherwise in the credit line; if the material is not included under the Creative Commons license, users will need to obtain permission from the license holder to reproduce the material. To view a copy of this license, visit http://creativecommons.org/licenses/by/4.0/

(C) The Author(s) 2016 